Ann. Génét. Sél. anim., I969, 1 (I), 9-13.

\title{
DEUX CAS D'INTERSEXUALITÉ CHROMOSOMIQUE CHEZ LE MOUTON (Ovis aries L.)
}

Françoise HULOT et P.-C. POPESCO

Station centrale de Génétique animale, Centre national de Recherches zootechniques, 78 -Jouy-en-Josas

Institut national de la Recherche agronomique

Institut de Recherches zootechniques, 63, rue du $D^{r}$-Staicovici, Bucarest, Roumanie

\section{SOMMAIRE}

Une analyse chromosomique, effectuée sur cultures sanguines de deux moutons anatomiquement intersexués, révèle, pour chacun d'eux, une juxtaposition de cellules mâles et femelles. L'un, au moins, de ces deux intersexués, serait un freemartin.

\section{INTRODUCTION}

Le nombre de chromosomes du mouton a été longtemps controversé (HULOT et LAUVERGNE, I967). Tous les auteurs s'accordent maintenant sur le nombre caractéristique de $2 n=5 .+$.

Parmi les autosomes, 6 apparaissent métacentriques et de grande taille, 46 sont télocentriques et peuvent être groupés en 23 paires de taille régulièrement décroissante. La position de l'hétérosome $\mathrm{X}$, parmi les télocentriques, n'est pas encore rigoureusement définie. Si certains l'identifient au plus petit (BORLAND, I964) ou à l'un des moyens (GERNEKE, I967), BRUÈRE et MCLAREN (I967) dans leur idiogramme, le placent en tête des télocentriques, ce qui corrobore l'interprétation de McFEe et al. (I965). Par contre, le chromosome $\mathrm{Y}$, de petite taille, métacentrique ou submétacentrique, est très caractéristique.

Jusqu'à présent, c'est surtout le caryotype de l'animal normal qui a fait l'objet d'études approfondies; les recherches cytogénétiques concernant les anomalies sexuelles, restent encore rares.

Le but de ce travail est de définir le sexe chromosomique de deux moutons intersexués. 


\section{MATÉRIEL ETT TECHNIQUE}

L'un des deux intersexués (A), de race Southdown ( ${ }^{1}$ ), né en janvier 1965, fait partie d'une portée de triplés qui comprend en outre une femelle normale et un mâle qui n'a survécu que quelques jours. L'examen de ses organes génitaux externes révèle un méat urinaire normalement . situé dans la vulve. Cet animal possède en outre un pénis vestigial et un instinct mâle développé.

L'autre intersexué (B) Southdown $\times$ Ravat $\left({ }^{2}\right)$ est né simple en mars 1965 . Il présente une vulve normale, un vagin réduit et un clitoris hypertrophié.

L'étude des chromosomes a été réalisée à partir de cultures de sang total, selon la microtechnique de DE Grovchy et al. (1964). Les milieux TC 199 ou NCTC ( $\left.{ }^{3}\right)$ ont été utilisés en présence soit du sérum de l'animal concerné, soit du sérum de veau.

L'observation des préparations microscopiques s'est limitée à l'identification de l'hétérosome $\mathrm{Y}$, permettant ainsi la détermination du sexe chromosomique des cellules en métaphases. Nous avons photographié environ 20 p. 100 des figures mitotiques afin de procéder à un comptage chromosomique rigoureux.

\section{RÉSULTATS}

Chez ces 2 animaux, présentant des caractères sexuels ambigus, nous observons à la fois des cellules sanguines XX et XY (tableau I).

\section{TABLEAU I}

Dénombrement des cellules $X X$ et $X Y$ chez 2 moutons intersexués

\begin{tabular}{|c|c|c|c|c|c|c|c|c|c|}
\hline \multirow{3}{*}{ ANIMAUX } & \multicolumn{4}{|c|}{$\begin{array}{l}\text { CELIULES FXAMINGES } \\
\text { AU MICROSCOPE }\end{array}$} & \multicolumn{5}{|c|}{ CELLULES PHOTOGRAPHIŚES } \\
\hline & \multirow{2}{*}{$\begin{array}{l}\text { Effectif } \\
\text { total }\end{array}$} & \multirow{2}{*}{$\mathrm{xx}$} & \multirow{2}{*}{$\mathrm{XY}$} & \multirow{2}{*}{$\% \mathrm{XY}$} & \multirow{2}{*}{$\begin{array}{l}\text { Effectif } \\
\text { total }\end{array}$} & \multicolumn{2}{|c|}{$2 n=54$} & \multicolumn{2}{|c|}{$2 n \neq 54$} \\
\hline & & & & & & $\mathrm{xx}$ & $\mathrm{XY}$ & $\mathrm{xx}$ & $\mathrm{XY}$ \\
\hline $\begin{array}{l}\text { A } \\
\text { Southdown }\end{array}$ & 77 & 74 & 3 & $4 \%$ & 14 & I I & 2 & $\begin{array}{c}I \\
(5 I)\end{array}$ & 0 \\
\hline $\begin{array}{l}\text { B } \\
\text { Southdown } \\
\times \\
\text { Ravat . . . }\end{array}$ & 97 & 34 & 63 & $65 \%$ & 24 & IO & I3 & $\begin{array}{c}\mathrm{I} \\
(52)\end{array}$ & o \\
\hline
\end{tabular}

Sur les clichés, nous avons pu vérifier que moins de ro p. Ioo des cellules $\mathrm{XX}$ sont incomplètes et que les cellules dans lesquelles est observé un chromosome $\mathrm{Y}$, comportent 54 chromosomes ce qui exclut pour ce dernier groupe l'éventualité d'une formule XXY.

(1) Charles Ecomard, Élevage de la Guérinière, Saint-Même-le-Tenu, 44.

(2) Élevage de Aurine Iréon, Durtol, 63.

(3) Milieu 199 de Morgan J. F., Mokton H. T., Parker P. C., I950. Milieu NCTC rog de McQunken W. T., Evans V. I., Earle I. R., I957; Labotatoire Eurobio, Paris. 


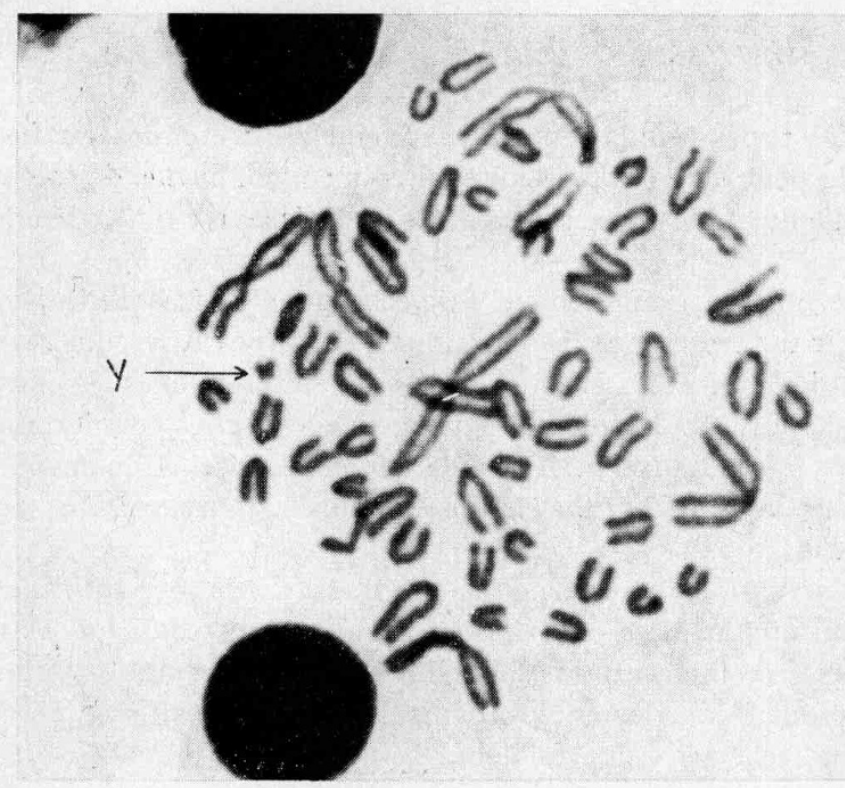

Fig. I. - Intersexué Southdown. Figure de métaphase $(X Y)$.

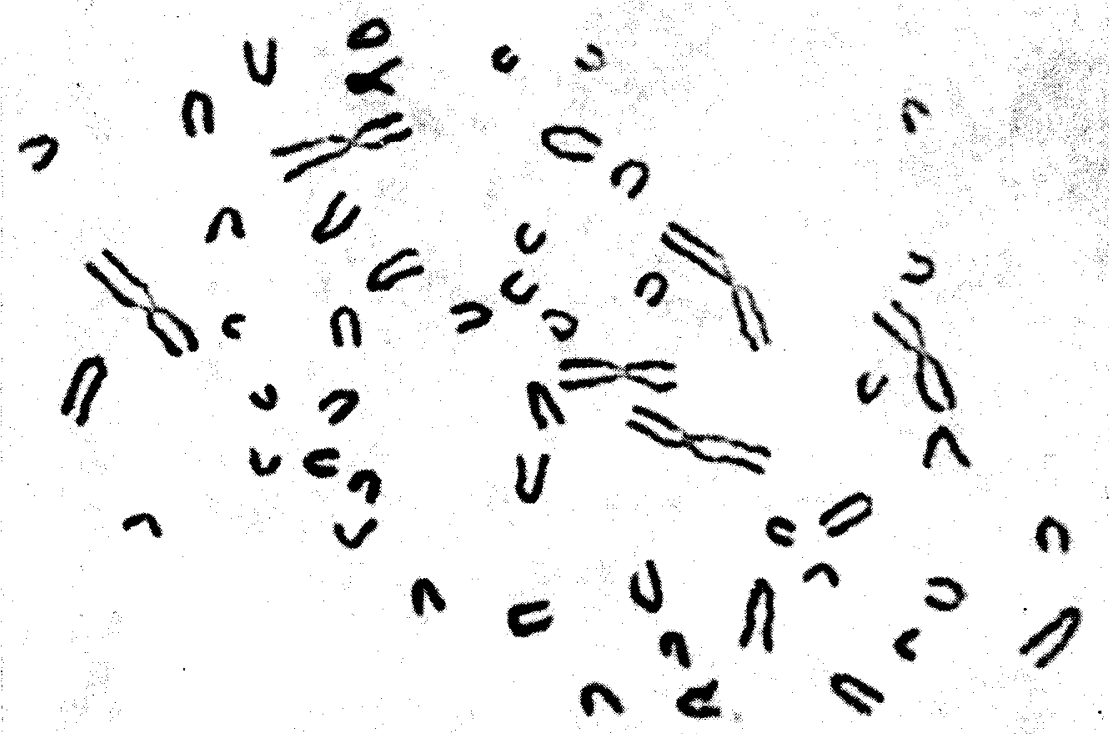

FIG. 2. - Intersexué Southdown $\times$ Ravat. Figure de métaphase $(X X)$. 


\section{DISCUSSION - CONCLUSION}

La juxtaposition des types cellulaires mâles et femelles a été notée dans de nombreuses espèces; elle peut être l'expression, soit d'un phénomène de freemartinisme, soit d'un dérèglement des mécanismes de la méiose ou de la fécondation.

En ce qui concerne plus particulièrement l'espèce ovine, le freemartinisme a été testé indirectement par la présence de mosaïques érythrocytaires qui se rencontreraient chez $0,8 \%$ des jumeaux issus de grossesses hétérosexuelles (STORMONT et al., I953; SLEE, I963), puis par la tolérance mutuelle de greffes ectodermiques (MOORE et Rowson, I958; SLEE, I963). Il a été démontré anatomiquement par l'existence d'anastomoses vasculaires allantoïdo-choriennes (ROTERMUND, I930; AIEXANDER et Williams, I964).

Par la suite, les observations caryologiques de GERNEke (I965, I967) sur la moelle osseuse, et de BruERE et MACNAB (I968) sur le sang, ont confirmé les possibilités de circulation croisée intra-utérine de cellules souches hématopoiétiques, et leur établissement définitif dans les deux organismes. Les autres tissus ne semblent pas affectés comme cela arrive parfois chez les bovins.

L'animal A qui avait un jumeau mâle est très probablement un freemartin. Le faible taux de cellules XY qu'il présente ne semble pas en contradiction avec cette hypothèse. En effet, BRUERE et MACNAB (I968) montrent une grande variation du pourcentage de leucocytes mâles, non seulement entre individus freemartins mais aussi entre cultures répétées sur un même individu.

En ce qui concerne l'intersexué $B$, né simple, le freemartinisme semble $a$ priori devoir être écarté, sauf si 1'on envisage un échange de tissu hématopoïétique in utero provenant d'un jumeau mâle éliminé précocement.

'Toutefois, la présence de leucocytes XY chez cet animal peut être le résultat de la fusion de deux zygotes ou de la fécondation dispermique d'ovules digyniques. Cette dernière hypothèse évoquée par GARTLER et al. (I962) dans l'espèce humaine, a été vérifiée par DE GROuchy et al. (I964) grâce à l'analyse des haptoglobines chez un sujet hermaphrodite humain. Par ailleurs, chez une chèvre, PADEH (I965) et chez un veau, DUNN et al., (I968) attribuent la juxtaposition de cellules sanguines XX et XY à l'un de ces deux mécanismes.

Afin de vérifier le bien-fondé de nos hypothèses sur l'origine de l'intersexualité de ces deux animaux, nous nous apprêtons à étendre nos investigations. Elles porteront, d'une part sur l'étude descriptive des organes génitaux (anatomique et histologique), d'autre part sur l'examen caryologique comparé de types cellulaires dérivant des trois feuillets embryonnaires primordiaux. Ces résultats complémentaires devraient apporter des éléments d'interprétation décisifs. 


\section{SUMMARY}

TWO CASES OF CHROMOSOMAL INTERSEXUALITY IN THE SHEEP

The aim of this study is to ascertain the chromosomal sex of two sheep which were anatomic intersexes, one being of the Southdown breed (A), the other being a Southdown X Ravat cross.

Microscopic examination of blood cell cultures (figures 1 and 2) showed that the two intersexes showed a reversal of male and female cells with a complete chromosomal complement. In animal A, XY cells constituted $4 \%$ of the total, while in animal B the percentage reached $65 \%$ (table 1).

Sheep $A$, which has a male twin, should be a freemartin.

Other mechanisms such as the fusion of two zygotes or the bi-spermic fertilization of a diploid ovum might be postulated for animal B which was born a single. These two hypotheses do not exclude however, the exchange of hematopoietic tissue in utero with a male twin aborted prematurely.

Anatomical and histological examination of the genital organs in addition to caryologic investigations of cell types of diverse embryonic origin ought to provide definitive results.

\section{RÉFÉRENCES BIBLIOGRAPHIQUES}

Alexander G. et Williams D., 1964. Ovine freemartins. Nature, (Lond.), 201, r296-I 298.

BORLAND R., x964. The chromosomes of domestic sheep. J. Hered., 55, 6r-64.

BRUERE A. N., MCLAREN R. D., r967. The idiogram of the sheep with particular reference to secondary constrictions. Canad. J. Genet. Cytol., 9, 543-553.

Brutere A. N., Macnab J., I968. A cytogenetical investigation of six intersex sheep, shown to be Freemartins. Res, veter. Sci., 9, I’0-180.

DUNN H. O., KENNEY R. M., LEIN D. H., rg68. XX/XY chimerism in a bovine true hermaphrodite $=$ an insight into the understanding of freemartinism. Cytogenetics, 7, 390-402.

MCFee A. F., BANNer M. W., MuRPhreE W. I., r965. Chromosome analysis of periferal leucocytes of the sheep. J. anim. Sci., 24, 55I-554.

Gartler S. M., WAXMaN S. H., GibletT F., r962. An XX/XY human hermaphrodite resulting from double fertilization. Proc. nation. Acad. Sci., (Paris), 48, 332-335.

GERNEkE W. H., x965. Chromosomal evidence of the freemartin condition in sheep. J. S. Afr. veter. med. Ass., 36, 99-104.

GERNERE W. H., 1967. Cytogenetic investigations on normal and malformed animals with special reference to intersexes. Onderstepoort $J$. veter. Res., 34, 219-300.

Grouchy J. DE, Moullec J., Salmon C. H., Josso N., Frezal J., Lamy M., ig64. Hermaphrodisme avec caryotype $\mathrm{XX} / \mathrm{XY}$. Étude génétique d'un cas. Ann. Génét., 7, 25-30.

Grouchy J. DE, Roubin M., PAssage E., I964. Microtechnique pour l'étude des chromosomes humains à partir d'une culture de leucocytes sanguins. Ann. Génét., 7, 45 .

Hulor Françoise et I,Auvergne J.-J., I967. L.es chromosomes des Ruminants. Ann. Génét., 10, 86-97.

MOore N. W. et Rowson I. E. A., I958. Freemartins in sheep. Nature, (Lond.), 182, I754-I755.

PaDeH B., Wysoki M., Ayalon N., Soller M., 1965. An XX/XY hermaphrodite in the goat. Israël J. med. Sci, 1, roo8-гог2.

Rotermund H., r930. Über Zwillingsfruchtsäcke kleiner Wiederkäuer. Morph. Jb., Leipzig, $64, x 76$.

SLEE J., I963. Immunological tolerance between littermates in sheep. Nature, (Iond.), 200, $654-656$.

Stormont C., WEIR W. C., et LANE I. I.., I953. Erythrocyte mosaicism in a pair of sheep twins. Science, N. Y., 118, $695-696$. 